\title{
Stabilization of Boron Carbide via Silicon doping
}

J.E. Proctor ${ }^{1,2, *}$, V. Bhakhri ${ }^{3}$, R. Hao ${ }^{3}$, T.J. Prior ${ }^{4}$, T. Scheler ${ }^{5}$, E. Gregoryanz ${ }^{5}$, M. Chhowalla ${ }^{6}$ and F. Giuliani ${ }^{3, *}$

${ }^{1}$ Department of Physics and Mathematics, University of Hull, Hull HU6 7RX, United Kingdom ${ }^{2}$ Joule Physics Laboratory, School of Computing, Science and Engineering, University of Salford, Manchester M5 4WT, United Kingdom

${ }^{3}$ Department of Materials Science, Imperial College London, London SW7 2AZ, United Kingdom

${ }^{4}$ Department of Chemistry, University of Hull, Hull HU6 7RX, United Kingdom

${ }^{5}$ School of Physics and Centre for Science at Extreme Conditions, University of Edinburgh, Edinburgh EH9 3JZ, United Kingdom

${ }^{6}$ Materials Science and Engineering, Rutgers University, Piscataway, New Jersey 08854, USA Boron carbide is one of the lightest and hardest ceramics, but its applications are limited by its poor stability against a partial phase separation into separate boron and carbon. Phase separation is observed under high non-hydrostatic stress (both static and dynamic), resulting in amorphization. The phase separation is thought to occur in just one of the many naturally occurring polytypes in the material, and this raises the possibility of doping the boron carbide to eliminate this polytype. In this work, we have synthesized boron carbide doped with silicon. We have conducted a series of characterizations (transmission electron microscopy, scanning electron microscopy, Raman spectroscopy and X-ray diffraction) on pure and silicon-doped boron carbide following static compression to $50 \mathrm{GPa}$ non-hydrostatic pressure. We find that the level of amorphization under static non-hydrostatic pressure is drastically reduced by the silicon-doping.

Keywords: Boron carbide, high pressure, amorphization, ceramics 


\section{Introduction}

Lightweight, impact-resistant materials are needed for applications such as the aerospace industry, where protection from space debris is required, and ballistic armour. In both cases high velocity impacts occur and the drive to reduce weight is huge. The most widely used impact-resistant ceramics are boron carbide $\left(\mathrm{B}_{4} \mathrm{C}\right)$, silicon carbide $(\mathrm{SiC})$, and alumina $\left(\mathrm{Al}_{2} \mathrm{O}_{3}\right)$. Boron carbide is the lightest and has potential to be the most effective. It possesses extreme hardness ( $45 \mathrm{GPa}$, surpassed only by diamond and cubic boron nitride) and higher Hugoniot Elastic Limit than any other ceramic material by a factor of $2(17-20 \mathrm{GPa})^{1}$. However it unexpectedly fragments under high-velocity impacts due to the formation of thin amorphous bands in the material ${ }^{2,3}$.

It was shown that the mechanism of failure of boron carbide against high velocity impacts also occurs in static high-pressure experiments using the diamond anvil high-pressure cell (DAC), provided the applied pressure is non-hydrostatic ${ }^{4}$. Raman spectroscopy ${ }^{4}$ and electron microscopy $\mathrm{y}^{2,4}$ have indicated that the failure of boron carbide is due to irreversible localized pressure-induced amorphization - the formation of narrow amorphous bands (<10 $\mathrm{nm}$ dia.) while most of the sample remains crystalline. Some limited amorphization was observed following static pressure-treatment to $25 \mathrm{GPa}$, with widespread formation of amorphous bands following pressurization to $35 \mathrm{GPa}^{4}$.

Pressure-induced amorphization is an important and widely studied phenomenon, observed in materials of importance in geology and planetary science ${ }^{5,6,7}$. It usually occurs when a phase transition between two crystal structures of an element / compound is kinetically inhibited, and in some cases when a dissociation of a compound into two separate compounds is kinetically inhibited $^{8}$. The pressure-induced amorphization of boron carbide is a very unusual case because it occurs due to a kinetically frustrated phase separation into separate elements. 
The amorphization of boron carbide is also unusual because the transition is irreversible and localized to $2-10 \mathrm{~nm}$ dia. bands. Therefore, the amorphous regions have been characterized using transmission electron microscopy (TEM) and Raman spectroscopy ${ }^{2,4}$ instead of the normal technique ${ }^{9,10}$ of X-ray diffraction. Due to the extremely localized nature of the amorphization process in boron carbide (TEM data ${ }^{2,4}$ shows that most of the material remains crystalline), the $\mathrm{X}$ ray diffraction signal from an amorphous area of boron carbide produced by static or dynamic pressure treatment is always convoluted with a signal orders of magnitude larger from remaining crystalline material.

In recent years, the mechanism for the pressure-induced amorphization of boron carbide causing its failure against non-hydrostatic stress has been understood on an atomic level in terms of its crystal structure. Boron carbide's structure consists of 12-atom icosahedra linked by 3-atom chains (figure 1). Due to the similarity in atomic volume between boron and carbon, it is difficult to determine which sites are occupied by which atoms. 


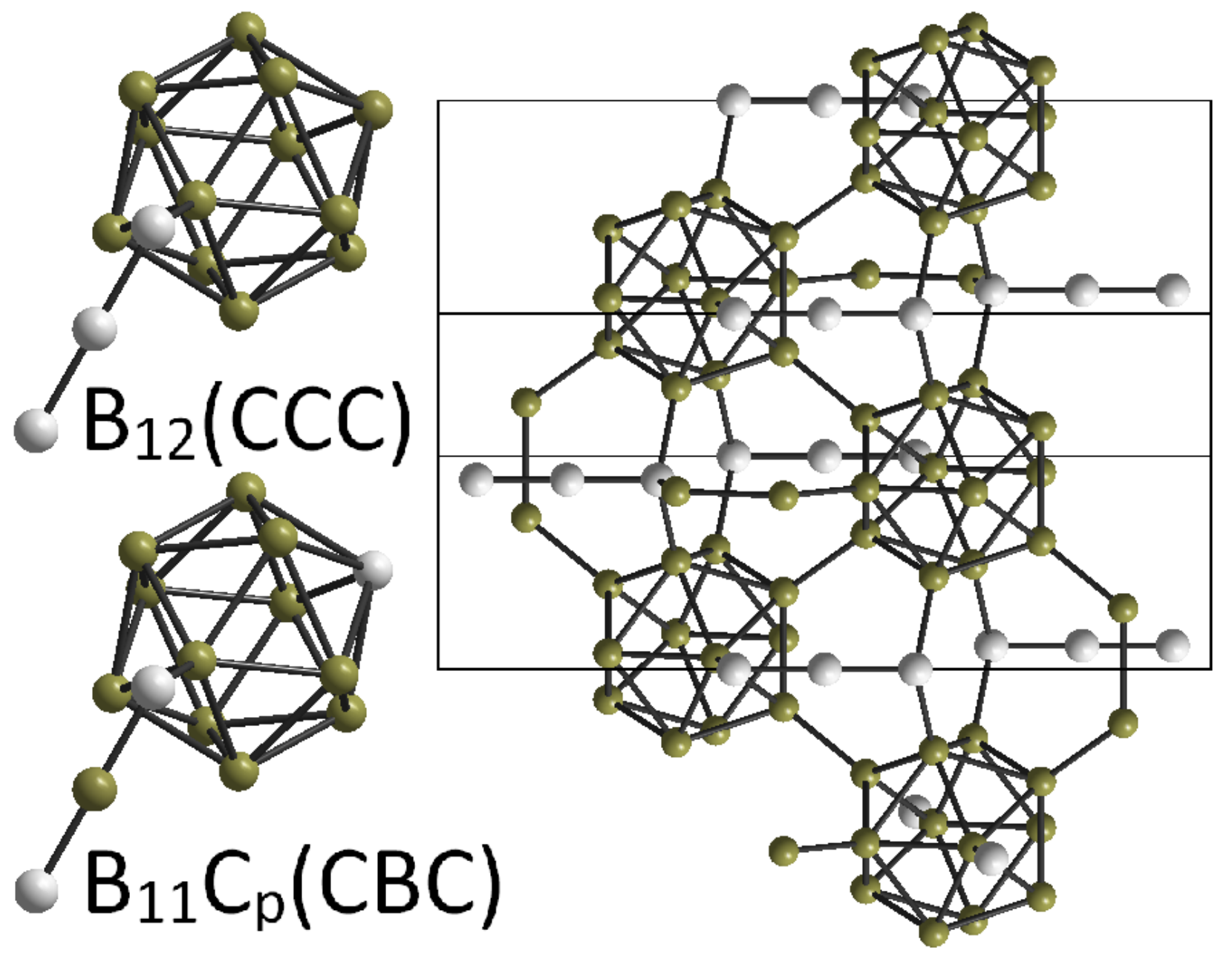

Fig. 1. Illustration of the crystal structure of boron carbide, consisting of 12 -atom icosahedra linked by 3 atom chains. Due to the similar atomic volumes of boron (green) and carbon (white), different arrangements (polytypes) of boron and carbon atoms within the icosahedra and chains are possible ${ }^{3}$. Two examples of polytypes thought to exist in pure boron carbide ${ }^{3,11}$ are shown on the left. It is believed that the existence of the $\mathrm{B}_{12}(\mathrm{CCC})$ polytype causes the pressure-induced amorphization process in pure boron carbide, due to the spatial proximity of the carbon atoms allowing the formation of small islands of amorphous carbon under stress. The diagram on the right shows how the icosahedra and chains fit together to form the boron carbide lattice, and how for the $\mathrm{B}_{12}(\mathrm{CCC})$ polytype (shown) this results in the close proximity of the carbon atoms.

Experimental studies aimed at elucidating this have produced conflicting results (reviewed in ref. 3). However, DFT calculations showed that, due to the similar atomic volumes of carbon and boron, differences in atomic arrangement cause changes to the Gibbs free energy of the material 
that are negligible compared to the thermal energies available during synthesis ${ }^{11}$. Variation in lattice parameters as a function of carbon atom location is also negligible.

It is therefore likely that different possible arrangements of boron and carbon atoms exist in pure boron carbide. These can be thought of as different polytypes, and described using notation such as $\mathrm{B}_{11} \mathrm{C}_{\mathrm{p}}(\mathrm{CBC}), \mathrm{B}_{12}(\mathrm{CBC})$ or $\mathrm{B}_{12}(\mathrm{CCC})$ (figure 1). The atoms in the chains are those in the brackets. The fact that the stoichiometry of crystalline boron carbide varies ${ }^{3}$ supports this hypothesis.

However, while the difference in Gibbs free energy between polytypes is negligible the energetic barrier to partial phase separation under pressure (origin of the observed amorphization) is predicted to vary strongly between the different polytypes. In particular, DFT calculations ${ }^{11}$ showed that the energetic barrier to partial phase separation under pressure is by far the lowest for the $\mathrm{B}_{12}(\mathrm{CCC})$ polytype. This is because the carbon atoms already sit in close proximity, so can form disordered graphitic/diamondlike islands with minimal movement.

This understanding leads to the conclusion that it may be possible to stabilize boron carbide against amorphization by doping with small quantities of a different element. Silicon is the obvious candidate for this, as it has a similar electronic structure to carbon. The addition of silicon can stabilize boron carbide in several ways. Firstly, it is likely to reduce the concentration of the $\mathrm{B}_{12}(\mathrm{CCC})$ polytype. DFT calculations ${ }^{12}$ have predicted that silicon-doping will drastically increase the difference in Gibbs Free Energy between the stable $\mathrm{B}_{11} \mathrm{C}_{\mathrm{p}}(\mathrm{CBC})$ polytype and the minority $\mathrm{B}_{12}(\mathrm{CCC})$ polytype, making formation of the latter polytype (the polytype responsible for the observed amorphization) energetically unfavourable when boron carbide is synthesized. Secondly, it should increase the energetic barrier to atom-swapping in the material, i.e. conversion between polytypes. DFT calculations ${ }^{11}$ have predicted that in pure boron carbide the energetic barrier to a conversion from the $\mathrm{B}_{11} \mathrm{C}_{\mathrm{p}}(\mathrm{CBC})$ polytype to the $\mathrm{B}_{12}(\mathrm{CCC})$ polytype becomes very low at high 
pressure. Addition of silicon should ameliorate this problem as it is believed that the silicon atoms preferentially sit at the poles of the icosahedra adjacent to the chains ${ }^{12}$. There is therefore no longer a carbon atom adjacent to the chain ready to move into the chain and form the $\mathrm{B}_{12}(\mathrm{CCC})$ polytype. Thirdly, the addition of silicon may ensure that the boron carbide compound remains the thermodynamically stable phase of the material (compared to phase-separated boron and carbon) to a much higher pressure. It is believed that pure boron carbide becomes thermodynamically unstable against a phase separation to elemental boron and carbon at just 7 GPa pressure ${ }^{11}$.

The synthesis of silicon-doped boron carbide presents an experimental challenge, as it is difficult to ensure the formation of silicon-doped boron carbide instead of boron carbide with elemental silicon, or silicon carbide with elemental boron. $\operatorname{Han}^{13}$ achieved an average silicon concentration of 0.38 at.\%. In this work, we synthesize boron carbide nanowires with much higher silicon content (0.81.6 at. \%), and perform static high-pressure experiments to experimentally test for the first time the stabilizing effect of silicon on the material.

\section{Methods}

Boron carbide nanowires doped with silicon were synthesized by the solid-liquid-solid method in which submicron boron powder (Sigma Aldrich, purity 99\%, initial particle size $0.82 \mu \mathrm{m}$ ), activated carbon (Norit America Inc., purity $\sim 99 \%$, initial particle size $5 \mu \mathrm{m}$ ) and silicon powder (Sigma Aldrich, purity $>99 \%$, initial particle size $4.27 \mu \mathrm{m}$ ) were used as the starting materials. A mixture (1 wt. \%) of Nickel Boride (NiB, Alfa Aesar, purity 99\%, initial particle size $500 \mu \mathrm{m})$, Nickel Diboride $\left(\mathrm{NiB}_{2}\right.$, Alfa Aesar, purity 99\%, initial particle size $\left.500 \mu \mathrm{m}\right)$ and Cobalt (Co, Alfa Aesar, purity $99.8 \%$, initial particle size $1.6 \mu \mathrm{m}$ ) was used as catalyst for the formation of the nanowires. The components and catalyst were mixed together and thoroughly ground using an agate mortar and pestle. The ratio was 1:1 B:C with $2 \mathrm{wt} \% \mathrm{Si}$. The reactant mixture was placed in a 10 
$\mathrm{mL}$ alumina combustion boat, which was inserted into a $99.8 \%$ dense alumina tube (length $70 \mathrm{~cm}$, inner diameter $6.35 \mathrm{~cm}$ ) in a clam shell furnace. The powder mixture was heated to $1150^{\circ} \mathrm{C}$ and held for 1 hour in argon at a pressure of $1 \mathrm{~atm}$.

TEM images along with a fast fourier transformation (FFT) confirming the single crystalline nature of such nanowires are shown in figure $2 \mathrm{a}-\mathrm{c}$. The chemical composition of the nanowires was obtained by electron energy loss spectroscopy (EELS) mapping as indicated by the colour plots in figure $2 \mathrm{~d}-\mathrm{f}$. It can be seen that the body of the nanowires consist of boron, carbon and silicon only and that the silicon is dispersed reasonably well throughout the entire nanowire segment. The chemical analysis was performed on approximately 100nm long segments of $\sim 50 \mathrm{~nm}$ diameter wires. Silicon content was confirmed with energy dispersive X-ray spectroscopy (EDX), and ranges from $0.8-1.6$ at. $\%$. The nanowires are believed to grow via the solid-liquid-solid mechanism: First, the solid precursors mix with the catalysts to form a low melting point liquid. This is followed by further precursor dissolving into the catalyst as the sticking coefficient is higher than the solid leading to supersaturation. Finally excess product is precipitated from catalyst in the form of nanowires. A more detailed discussion of the growth mechanism and kinetics is given in ref. 14 .

Although in some cases catalyst particles can be observed at the ends of the nanowires after growth, no evidence of catalyst particles was observed in the X-ray diffraction patterns or Raman spectra. Therefore, in terms of volume and mass, the catalyst particles make up a negligible proportion of the sample (each $\sim 1 \mathrm{~mm}$ long wire grows from a single catalyst particle). Furthermore it should be noted that we did not detect silicon carbide in the silicon doped sample which is the major advantage of this synthesis technique. 

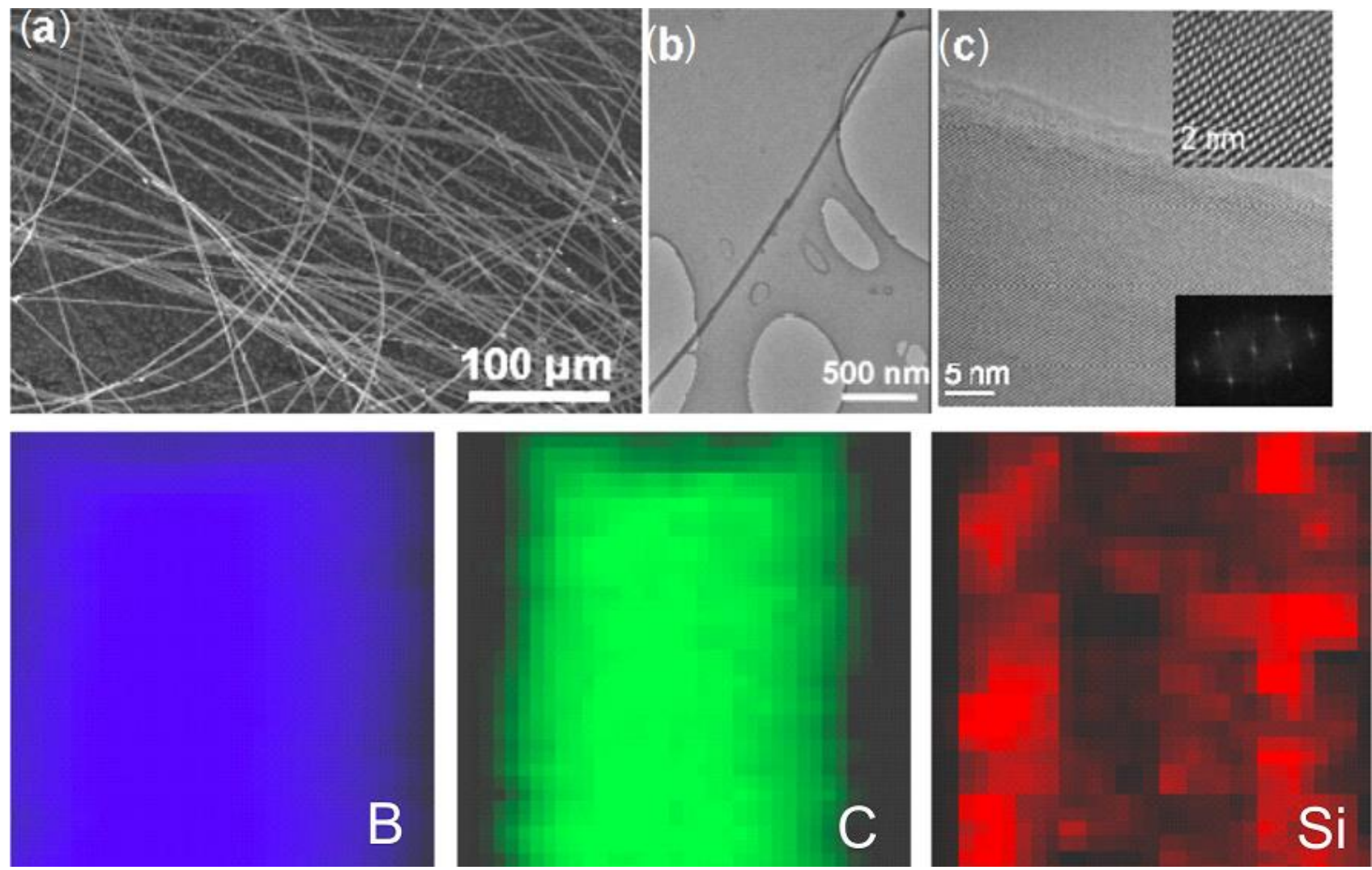

Fig. 2. Upper panel: TEM images (a, b, c, top inset of c) and corresponding FFT (bottom inset of (c)) of silicon-doped boron carbide nanowires as-produced. Lower panel: Chemical mapping of as-produced silicondoped boron carbide nanowires performed with EELS. The element corresponding to each color is indicated in the lower right of each EELS plot.

The nanowires were ball milled for one hour in an argon atmosphere and then consolidated by spark plasma sintering (SPS). The samples were densified at $2100^{\circ} \mathrm{C}$ under $50 \mathrm{MPa}$ for $20 \mathrm{~min}$. No sintering aids were added to the powder. The densities of the samples measured by the Archimedes method $\left(2.5 \mathrm{gcm}^{-3} \pm 0.1\right)$ were in agreement with the theoretical value $\left(2.52 \mathrm{gcm}^{-3}\right)$.

The pure boron carbide samples used were powder produced by H.C. Starck, Grade HP and purchased via Sigma Aldrich.

Pressure-treatment under static non-hydrostatic conditions was performed by compressing the material in rhenium-gasketed diamond anvil cells (DACs) $(250 \mu \mathrm{m}$ dia. culets). Pressure was 
measured using the standard techniques of performing X-ray diffraction on a small grain of tantalum in the sample chamber, or by performing photoluminescence on a ruby microcrystal in the sample chamber. To ensure non-hydrostatic conditions the rest of the sample chamber was filled with the boron carbide sample. No pressure-transmitting medium was employed.

TEM lamella were produced from samples after high pressure treatment using focussed ion beam (FIB) milling following the in situ liftout procedure, on a FEI, Helios dual beam FIB. A final low energy polish at $2 \mathrm{kV}$ and $28 \mathrm{pA}$ was then carried out. Imaging and spectroscopy were performed on a monochromated and image corrected FEI, Titan operating at $300 \mathrm{kV}$.

Raman spectra were collected in the backscattering geometry using two Raman microscopes, a Renishaw 1000 instrument and a custom-constructed insrument. In both cases, the laser beam $(514 / 532 \mathrm{~nm})$ was focussed to a spot size of $\approx 1 \mu \mathrm{m}$ on the sample using a $50 \mathrm{x}$ objective lens and the laser power reaching the sample was significantly lower than $5 \mathrm{~mW}$. We found that using a much higher incident laser power caused localized damage to the sample so worked carefully to avoid this throughout. Raman spectra were collected using 1200 lines per inch diffraction gratings, nitrogen and Peltier-cooled CCD detectors. TEM and Raman data were collected from different areas of the samples.

Powder X-ray diffraction data was collected at beamline I15 at the Diamond Light Source, UK. The incident X-ray wavelength was $0.4133 \AA$ and the beam size was approximately $50 \mu \mathrm{m}$. The diffraction data were collected on a mar345 image plate and the 2-dimensional diffraction images (60 second exposure time) were integrated using Fit2D ${ }^{15}$ to give standard diffraction profiles. Xray diffraction data were analysed using a Pawley structureless fitting procedure within the GSAS ${ }^{16}$ suite of programs to determine the peak positions and peak intensities. Instrumental peak shape broadening was estimated from the diffraction data collected from a silicon standard at $0 \mathrm{GPa}$. 


\section{Results}

Pure boron carbide has been shown by other authors to undergo a localized irreversible amorphization process after pressure-treatment to $25 \mathrm{GPa}$ non-hydrostatic pressure in the $\mathrm{DAC}^{4}$. In this work, we therefore compressed several samples of both pure and silicon-doped boron carbide to $50 \mathrm{GPa}$ non-hydrostatic pressure in the DAC to evaluate the stability of both materials.

Our TEM study of pure boron carbide after pressure-treatment to $50 \mathrm{GPa}$ revealed the presence of localized amorphous areas and widespread microcracking, as observed by other authors following both shock compression ${ }^{2}$ and non-hydrostatic compression in the DAC ${ }^{4}$. In contrast, our TEM study of silicon-doped boron carbide after pressure-treatment did not reveal amorphous bands - the material remained entirely in its (poly)crystalline state. Figure 3 shows representative TEM images of pure and silicon-doped boron carbide after non-hydrostatic pressure-treatment to $50 \mathrm{GPa}$. Fast Fourier Transform (FFT) images are included to verify the crystalline (or non-crystalline) nature of different areas of the samples. See supplementary material for further images of both pure and silicon-doped material.

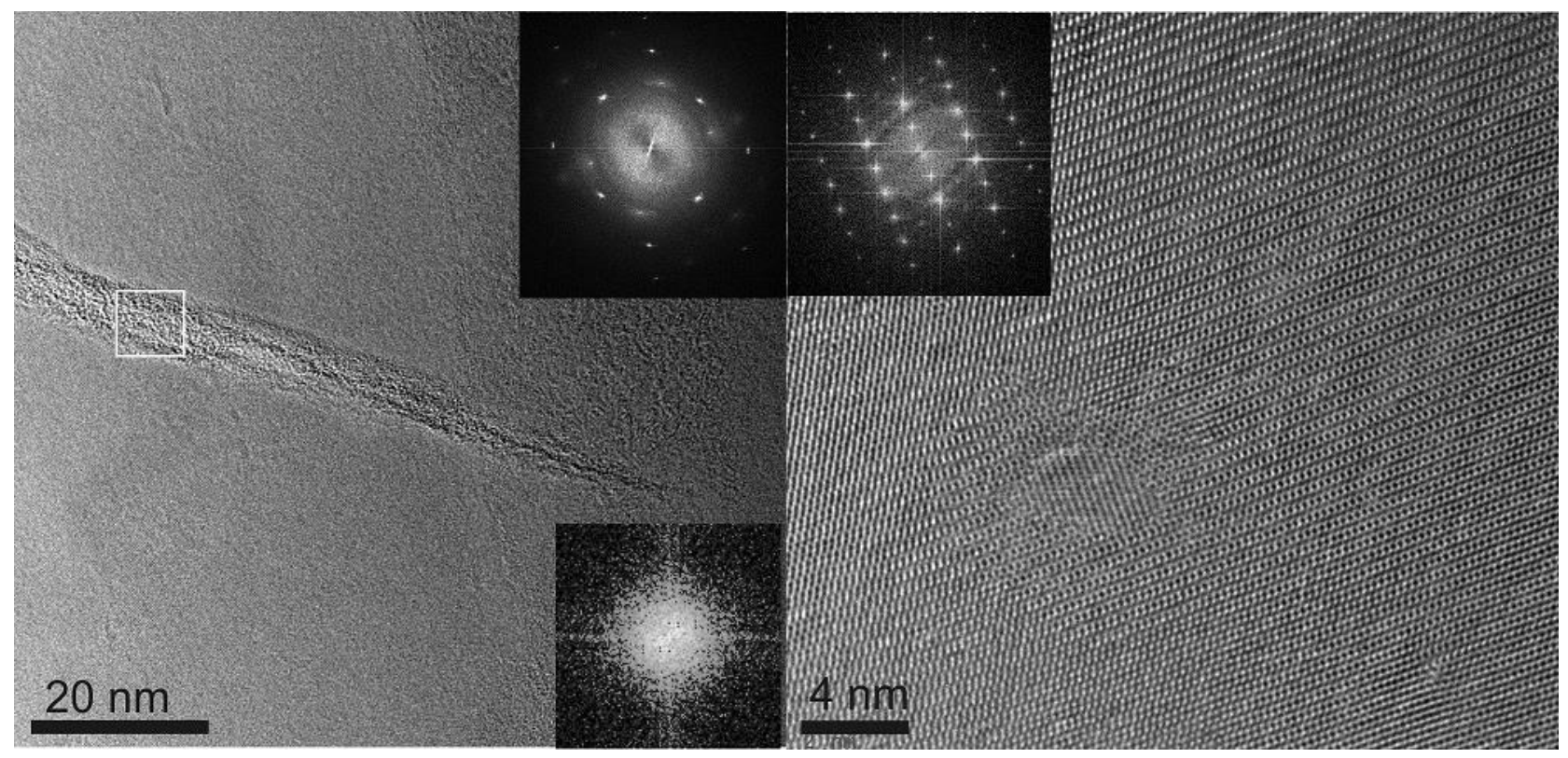


Fig. 3. TEM images of pure (left) and silicon-doped (right) boron carbide after pressure-treatment to 50 GPa. In the pure material (left), amorphous regions $2-10 \mathrm{~nm}$ in diameter were frequently observed, in agreement with the findings of other authors. The inset on the top right is an FFT of the whole image ( consistent with a [101] zone axis) while the inset on the bottom right is from the band where the box is marked and can be seen to be amorphous. In the silicon-doped material (right), we did not observe a single amorphous area - the material remained crystalline throughout. The inset on the top left is an FFT of the whole image (consistent with a (011) zone axis).

To evaluate the resilience of the materials on a macroscopic scale we took Scanning Electron Microscopy (SEM) images of cross-sections through the pressure-treated pure and silicon-doped material cut using a focussed ion beam. A large network of cracks is seen running across the pure material while the silicon doped material is crack free (figure 4).

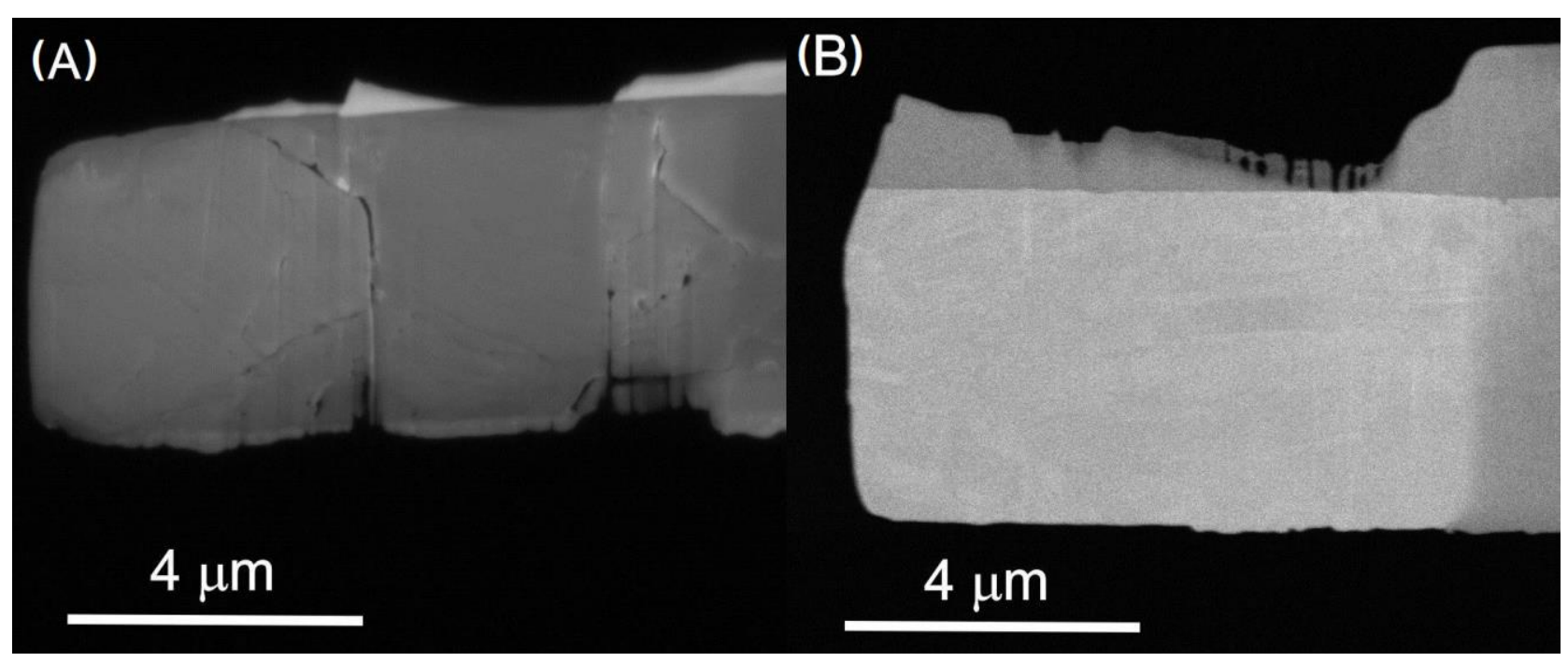

Fig. 4. SEM images taken during the final stages of TEM sample preparation by FIB of (A) pure boron carbide showing significant micro cracking and (B) silicon-doped boron carbide showing no cracking.

In our Raman spectroscopy experiments the contrast between pure and silicon-doped boron carbide was just as striking. After pressurizing both pure and silicon-doped boron carbide to 50 GPa nonhydrostatic pressure 10 Raman spectra of each sample from different locations on the sample surface were collected. In the pure material, the Raman peaks characteristic of amorphous boron 
carbide (particularly the strongest peak at ca. $1325 \mathrm{~cm}^{-1}$ ) appeared with significant intensity in 7 out of 10 spectra. In the silicon-doped material, these peaks appeared in only 1 in 10 spectra. Example Raman spectra are shown in figure 5 and further Raman spectra (collected before and after pressure-treatment) are shown in the supplementary materials.

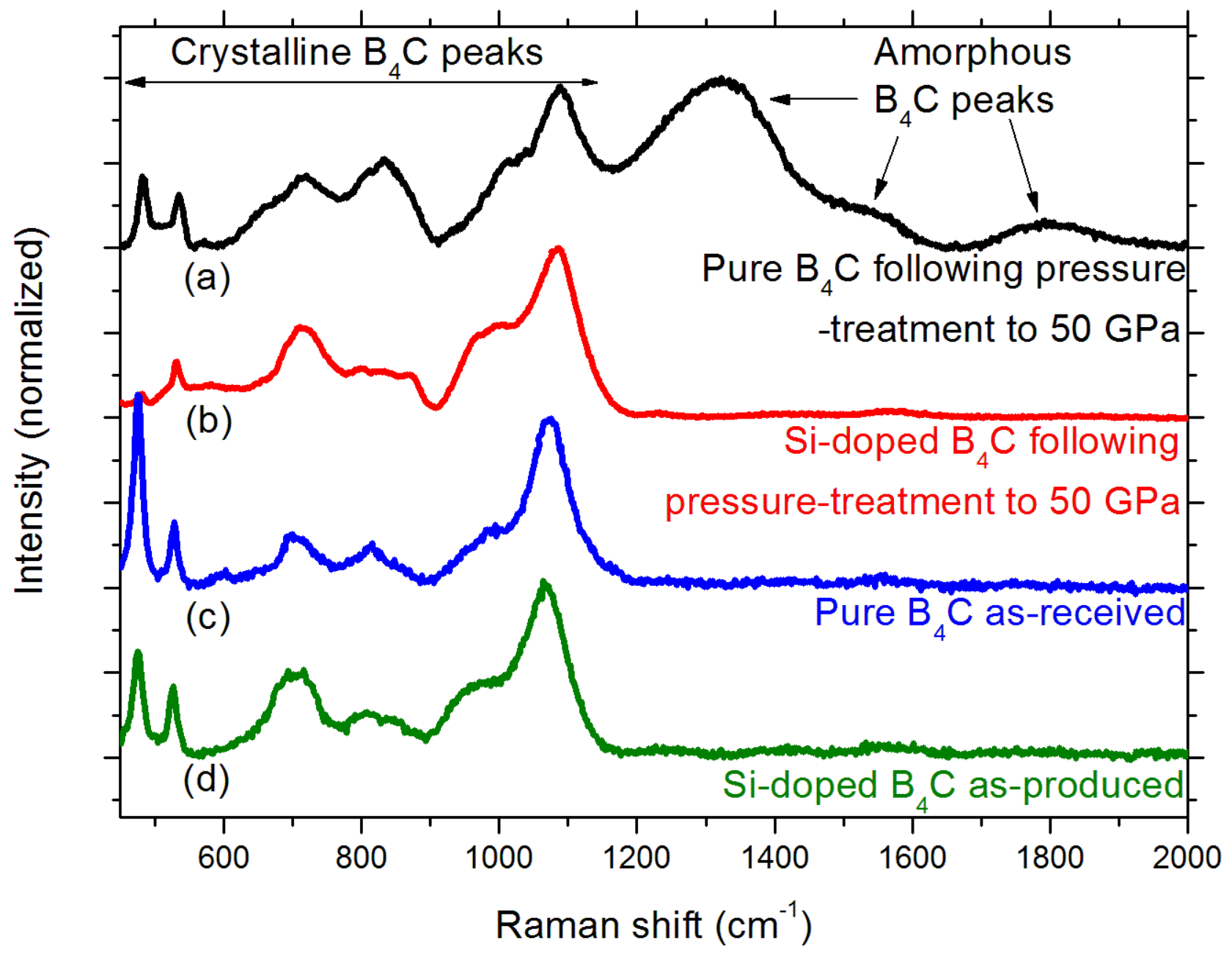

Fig. 5. Raman spectra of pure ((a) and (c)) and silicon-doped ((b) and (d)) boron carbide taken before and after pressure-treatment to $50 \mathrm{GPa}$. In all spectra collected before pressure treatment $((\mathrm{c})$ and $(\mathrm{d}))$ the crystalline boron carbide peaks from $450-1200 \mathrm{~cm}^{-1}$ are the strongest peaks present. Following pressuretreatment of the pure sample (a), peaks originating from amorphous boron carbide ${ }^{4}$ at ca. $1325 \mathrm{~cm}^{-1}$, $1520 \mathrm{~cm}^{-1}$ and $1810 \mathrm{~cm}^{-1}$ appear with significant intensity in $7 / 10$ of spectra collected. In the case of the silicon-doped sample (b), these peaks appeared in only $1 / 10$ of spectra collected. See supplementary material for further Raman spectra. 
We also performed an experiment in which we compressed silicon-doped boron carbide to much higher pressure, $67 \mathrm{GPa}$. Following this experiment, Raman spectra of the sample demonstrated widespread amorphization (figure 6). Further characterization of this sample was therefore not attempted.

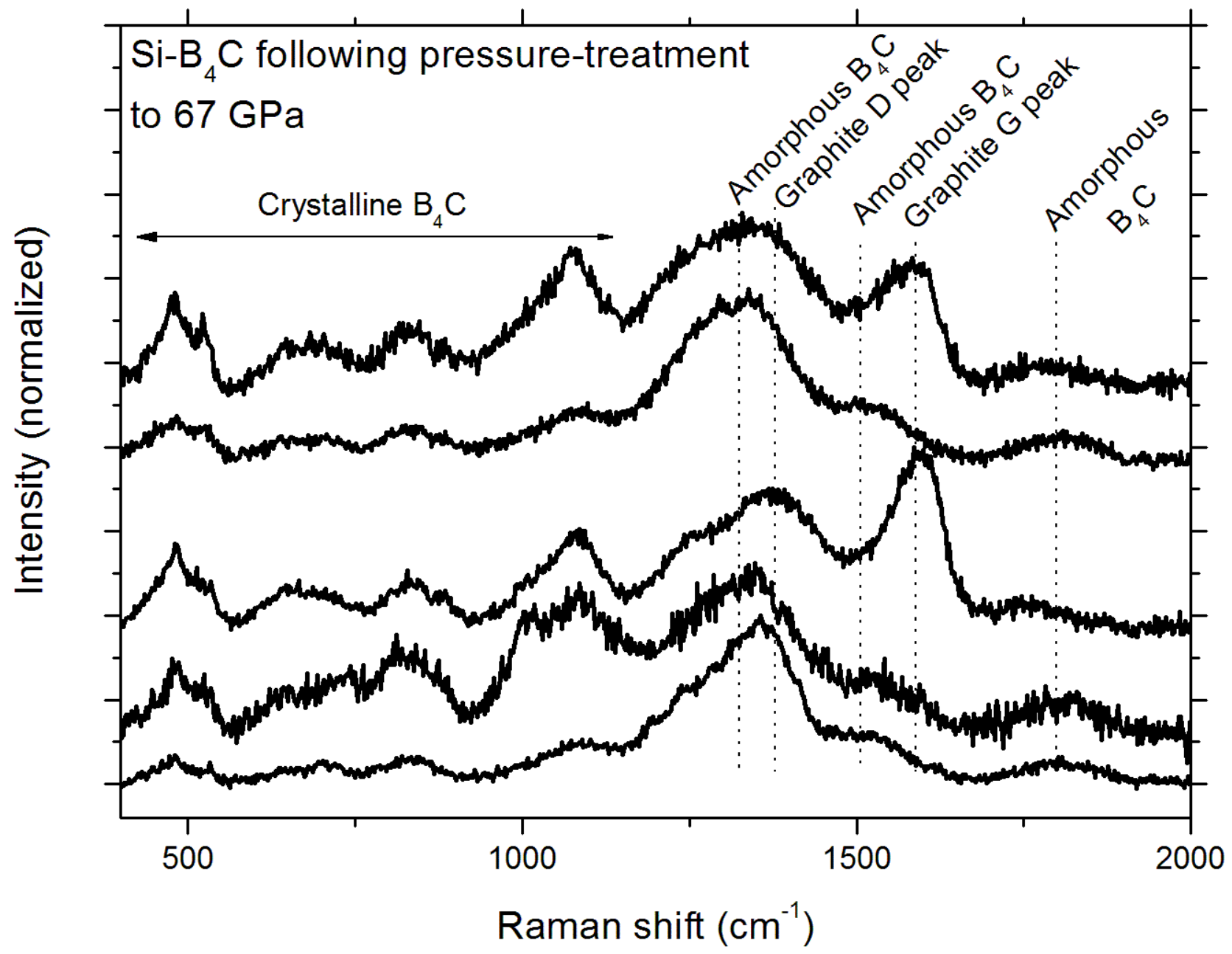

Fig. 6. Raman spectra of silicon-doped boron carbide after pressure-treatment to $67 \mathrm{GPa}$ collected from 5 different locations in the sample chamber. Crystalline $B_{4} C$ peaks are now weak and peaks from amorphous $\mathrm{B}_{4} \mathrm{C}$ and carbon now dominate. The peak at ca. $1580 \mathrm{~cm}^{-1}$ is the $\mathrm{G}$ peak of disordered carbon while the peak at ca. $1350 \mathrm{~cm}^{-1}$ contains contributions from the D peak of disordered carbon and the most intense Raman peak from amorphous boron carbide. 
Commercially available boron carbide always contains some graphite ${ }^{3,17,18}$, as expected from theory $^{11}$. Our Raman spectroscopy experiments revealed the presence of some graphite in both pure and silicon-doped samples as-received/produced. In some spectra, graphite Raman peaks ${ }^{19,20}$ at ca. $1340 \mathrm{~cm}^{-1}$ and ca. $1580 \mathrm{~cm}^{-1}$ were observed. Higher graphite content was found in the silicon-doped samples. We did not observe an increase in graphite content following pressure treatment in either sample, but Raman spectroscopy can only provide a qualitative measure of the graphitic / diamondlike nature of a disordered carbon sample. This is especially true for spectra such as those in fig. 6 where a number of peaks significantly overlap, preventing accurate fitting of relative intensities. A peak at frequency close to the graphite $\mathrm{G}$ peak $\left(\mathrm{ca} .1580 \mathrm{~cm}^{-1}\right)$ is observed even in samples of boron carbide that are believed to be single crystal (refs. 3, 4, and refs. therein). The origin of this peak is debated - potentially the formation of a boron carbide polytype with a CBB chain could also lead to the appearance of a Raman peak here ${ }^{3}$. For figure 5 we chose, for clarity, spectra that did not show graphite / CBB chain content, for spectra demonstrating the graphite / CBB chain content see supplementary material.

We also performed synchrotron X-ray powder diffraction on pure and silicon-doped boron carbide samples before and after pressure-treatment, and in-situ in the DAC at high pressure. Evidence of structural collapse under pressure was not found and (within experimental error) the compressibility of the material is not affected by the silicon-doping. The X-ray diffraction results do not conclusively demonstrate the presence of amorphous areas in the pure sample following pressuretreatment, as expected from the observation with TEM that most material remains crystalline and in agreement with other authors ${ }^{21}$. We did, however, observe X-ray diffraction peak broadening following pressure treatment that was greater in the pure sample than the silicon-doped sample, consistent with reduction in crystallite size (Scherrer broadening) due to the creation of amorphous 
areas in the pure sample observed in TEM. See supplementary material for further details of X-ray diffraction results.

\section{Discussion}

Other authors ${ }^{4}$ demonstrated that just $25 \mathrm{GPa}$ of non-hydrostatic pressure is required to induce the localized amorphization process that prevents pure boron carbide from withstanding high-velocity impacts. Here, we have confirmed the presence of widespread localized amorphization in pure boron carbide following pressure-treatment to $50 \mathrm{GPa}$, and demonstrated that there is virtually no amorphization in silicon-doped boron carbide following treatment to the same pressure - a significant improvement in stability compared to pure boron carbide. However, we found that a pressure of $67 \mathrm{GPa}$ did induce significant levels of amorphization even in the silicon-doped material. It is predicted theoretically that, in pure boron carbide, the energetic barrier to atomswapping (i.e. conversion between polytypes) and therefore amorphization reduces as a function of pressure $^{11}$. It is likely that this is also true to some extent even for silicon-doped boron carbideensuring that, if pressure is high enough, even silicon-doped boron carbide will amorphize.

It is important to note that the significant stabilizing effect of silicon that we have observed experimentally must be achieved through several different mechanisms, as proposed earlier. A reduction in $\mathrm{B}_{12}(\mathrm{CCC})$ content in the material as-synthesized due to the presence of silicon will undoubtedly play a role, but for the average silicon content in our samples (1.2 at. \%) we still expect a significant quantity of the $\mathrm{B}_{12}(\mathrm{CCC})$ polytype to form. Using results from DFT calculations of the Gibbs free energies of different polytypes in the pure ${ }^{11}$ and silicon-doped ${ }^{12}$ samples it is possible to predict the concentration of the different polytypes. We expect that a sample doped with 1.2 at. $\%$ silicon will exhibit $\mathrm{B}_{12}(\mathrm{CCC})$ polytype concentration $91 \%$ of that in pure boron carbide. We expect that in the synthesis temperature range of interest (1400 K to 2200 
$\mathrm{K}) \mathrm{B}_{12}(\mathrm{CCC})$ concentration will vary little with temperature, especially for the silicon-doped samples. In both cases we expect $\mathrm{B}_{12}(\mathrm{CCC})$ concentration to increase slightly as growth temperature is decreased. This is because, while this polytype has slightly higher Gibbs free energy than the stable polytype $B_{11} C_{p}(C B C)(\Delta G=0.03781 \mathrm{eV})$, the polytypes with even higher Gibbs free energy (e.g. $B_{11} C_{p}(C C B)$ with $\Delta G=0.07789 \mathrm{eV}$ ) are eliminated first as temperature is decreased with $\mathrm{B}_{11} \mathrm{C}_{\mathrm{p}}(\mathrm{CBC})$ and $\mathrm{B}_{12}(\mathrm{CCC})$ concentration both increasing to compensate. See supplementary material for further information on the expected effect of synthesis temperature on polytype distribution.

If our prediction that $\mathrm{B}_{12}(\mathrm{CCC})$ concentration in our silicon-doped samples is $91 \%$ of that in pure boron carbide is correct, then it is likely that the stabilizing effect observed of silicon-doping must occur through other mechanisms also, in addition to reducing the $\mathrm{B}_{12}(\mathrm{CCC})$ concentration upon synthesis. Most probable is that the addition of silicon inhibits formation of the $\mathrm{B}_{12}(\mathrm{CCC})$ polytype under pressure through atom-swapping, and increases the maximum pressure at which the boron carbide compound is thermodynamically stable against phase separation. Further theoretical studies would be useful to elucidate these issues.

Previous experimental ${ }^{2,4}$ and theoretical ${ }^{22}$ studies have demonstrated the necessity of a nonhydrostatic stress component to induce amorphization in pure boron carbide. Under impact (extremely non-hydrostatic conditions), only $\approx 23$ GPa pressure is required for the amorphization process $^{2}$. Under non-hydrostatic static compression in the DAC, limited amorphization was observed following treatment to $25 \mathrm{GPa}$ pressure and widespread amorphization following treatment to $35 \mathrm{GPa}^{4}$. Under quasi-hydrostatic conditions provided by an $\mathrm{NaCl}$ pressuretransmitting medium pure boron carbide was found to be stable to $50 \mathrm{GPa}$, the highest pressure reached in the study ${ }^{4}$. 
In both our study and ref. 4 the comparison between the Raman spectra and TEM images of pure boron carbide following pressure-treatment provides additional evidence regarding the role of nonhydrostatic stress in inducing pressure-induced amorphization. In the Raman spectra of pure boron carbide following pressure-treatment to $50 \mathrm{GPa}$ (fig. 5 in main text, fig. S2 and ref. 4) the crystalline peaks are often weaker than the amorphous peaks, indicating that areas comparable in diameter to the $\sim 1 \mu \mathrm{m}$ dia. focussed laser beam have amorphized. In contrast, the TEM images (fig. 3 in main text, figures S5 and S6) show most material remaining crystalline and only very narrow $(2-10 \mathrm{~nm})$ bands amorphizing.

In the Raman spectra of silicon-doped boron carbide following pressure-treatment to $50 \mathrm{GPa}$ (fig. 5 in main text and fig. S2) we observe the peaks from amorphous boron carbide in 1 in 10 spectra, while in the TEM images collected (fig. 3 in main text, figs. S5 and S6) we did not observe a single amorphous area of any size.

This is due to the fact that Raman spectroscopy of an opaque material such as boron carbide or graphite is a surface technique, probing only the first $\sim 50$ atomic layers. In contrast, the TEM images are from crossections of $\sim 10 \mu \mathrm{m}$ through the material. In our DAC experiments, nonhydrostatic stresses are much greater at the surfaces of the sample touching the diamond anvils, and therefore it is not inconsistent for there to be a weak amorphous signal on the surface detected using Raman spectroscopy but no amorphous areas detected in the bulk of the sample using TEM.

Pure boron carbide is not the only material in which the presence of a non-hydrostatic stress component is necessary to cause pressure-induced amorphization. For instance, non-hydrostatic conditions were also found to be necessary to observe pressure-induced amorphization in sulphur at room temperature ${ }^{23-25}$. 


\section{Conclusion}

In conclusion, we have synthesized silicon-doped boron carbide and performed a series of experiments (TEM, SEM, Raman spectroscopy and X-ray diffraction) demonstrating that the silicon-doping significantly suppresses the static pressure-induced amorphization previously reported for pure boron carbide. In a direct comparison between pure and silicon-doped boron carbide following treatment at $50 \mathrm{GPa}$ non-hydrostatic pressure we observe widespread amorphization in pure boron carbide and virtually no amorphization in silicon-doped boron carbide. Our results indicate that the static pressure required to induce widespread amorphization in boron carbide is approximately doubled as a result of doping with silicon, from $35 \mathrm{GPa}^{4}$ to $67 \mathrm{GPa}$.

It is now urgent to find ways to synthesize larger quantities of this new material to enable ballistic testing. If it is found that silicon-doping also suppresses the amorphization process under shock compression then silicon-doped boron carbide could significantly out-perform the current state-ofthe-art materials in terms of performance against high velocity impacts, while remaining the lightest major ceramic. Even a relatively small improvement in the performance of boron carbide against high velocity impacts would be of significant technological interest.

*Correspondence to: j.e.proctor@salford.ac.uk, f.giuliani@imperial.ac.uk

\section{Acknowledgements}

This work was funded by the Materials and Structures Science and Technology Centre through the UK Centre for Defence Enterprise under contract DSTLX 1000045292, and by the Engineering \& Physical Sciences Research Council of the United Kingdom through Grant number EP/F033605/1. X-ray diffraction data were collected at beamline I15 of the Diamond synchrotron in Didcot, UK (beamtime EE6833). Prof. Eduardo Saiz Gutierrez (Imperial College), Prof. Georg Mehl (University of Hull), Dr. John Loveday (University of Edinburgh) and Dr. Vladislav Domnich 
(Rutgers University) read the manuscript and provided helpful comments and suggestions. Prof. Matthew Halsall and Dr. Iain Crowe (University of Manchester) provided access to Raman spectroscopy apparatus at Manchester for some of the experiments, and Dr. Simon Macleod (Imperial College) loaned equipment for a preliminary set of experiments. We would also like to acknowledge the work and expertise of Nigel Parkin and Chris Lloyd at the University of Hull Chemistry Dept. workshop in building the high pressure cells used for most of the experiments presented here.

\section{References}

1. N. K. Bourne, Proc. R. Soc. London A 458, 2002 (1999).

2. M. Chen, J. W. McCauley and K. J. Hemker, Science 299, 1563 (2003).

3. V. Domnich, S. Reynaud, R. A. Haber and M. Chhowalla, J. Am. Ceram. Soc. 94, 3605 (2011). 4. X. Q. Yan, Z. Tang, L. Zhang, J. J. Guo, C. Q. Jin, Y. Zhang, T. Goto, J. W. McCauley and M. W. Chen, Phys. Rev. Lett. 102, 075505 (2009).

5. O. Mishima, L. D. Calvert and E. Whalley, Nature 310, 393 (1984).

6. R. J. Hemley, A. P. Jephcoat, H-K. Mao, L. C. Ming and M. H. Manghnani, Nature 334, 52 (1988).

7. S. K. Deb, M. Wilding, M. Somayazulu and P. F. McMillan, Nature 414, 528 (2001).

8. S. M. Sharma and S. K. Sikka, Prog. Mat. Sci. 40, 1 (1996).

9. M. Hanfland, J. E. Proctor, C. L. Guillaume, O. Degtyareva and E. Gregoryanz, Phys. Rev. Lett. 106, 095503 (2011).

10. C. Sanloup, E. Gregoryanz, O. Degtyareva and M. Hanfland, Phys. Rev. Lett. 100, 075701 (2008). 
11. G. Fanchini, J. W. McCauley and M. Chhowalla, Phys. Rev. Lett. 97, 035502 (2006).

12. G. Fanchini, D. E. Niesz, R. A. Haber, J. W. McCauley and M. Chhowalla, Advances in Ceramic Armor II, Ceram. Eng. \& Sci. Proc. 27, 7, 179 (2006).

13. W-Q. Han, Appl. Phys. Lett. 88, 133118 (2006).

14. V Gupta, Determination of structural changes and phase transformations in boron carbide by static and dynamic studies (Ph.D. Thesis), Rutgers University (2010).

15. A. P. Hammersley, S. O. Svensson, M. Hanfland, A. N. Fitch and D. Hausermann, High Press. Res. 14, 235 (1996).

16. A. C. Larson and R. B. Von Dreele, "General Structure Analysis System (GSAS)", Los Alamos National Laboratory Report LAUR 86-748 (1994).

17. F. Thévenot, J. Eur. Ceram. Soc. 6, 205 (1990).

18. M. Chen, J.W. McCauley, J.C. LaSalvia and K.J. Hemker, J. Am. Ceram. Soc. 88, 1935 (2005).

19. A. C. Ferrari and J. Robertson, Phys. Rev. B 61, 14095 (2000).

20. X. Q. Yan, W. J. Li, T. Goto and M. W. Chen, Appl. Phys. Lett. 88, 131905 (2006).

21. D. P. Dandekar, J. A. Ciezak and M. Somayazulu, Proceedings of the 26th Army Science Conference, Orlando, Florida (Dec. 2008), US Army DTIC report.

22. S. Aryal, P. Rulis and W. Y. Ching, Phys. Rev. B 84, 184112 (2011).

23. Y. Akahama, M. Kobayashi and H. Kawamura, Phys. Rev. B 48, 6862 (1993).

24. H. Luo and A. L. Ruoff, Phys. Rev. B 48, 569 (1993).

25. C. Hejny, L. F. Lundegaard, S. Falconi, M. I. McMahon and M. Hanfland, Phys. Rev. B 71, $020101(2005)$. 
26. T. Fujii, Y. Mori, H. Hyodo and K. Kimura, J. Phys. Conf. Ser. 214, 012011 (2010). 


\section{Supplementary materials}

\section{Raman spectra of pure and silicon-doped boron carbide as received/produced}
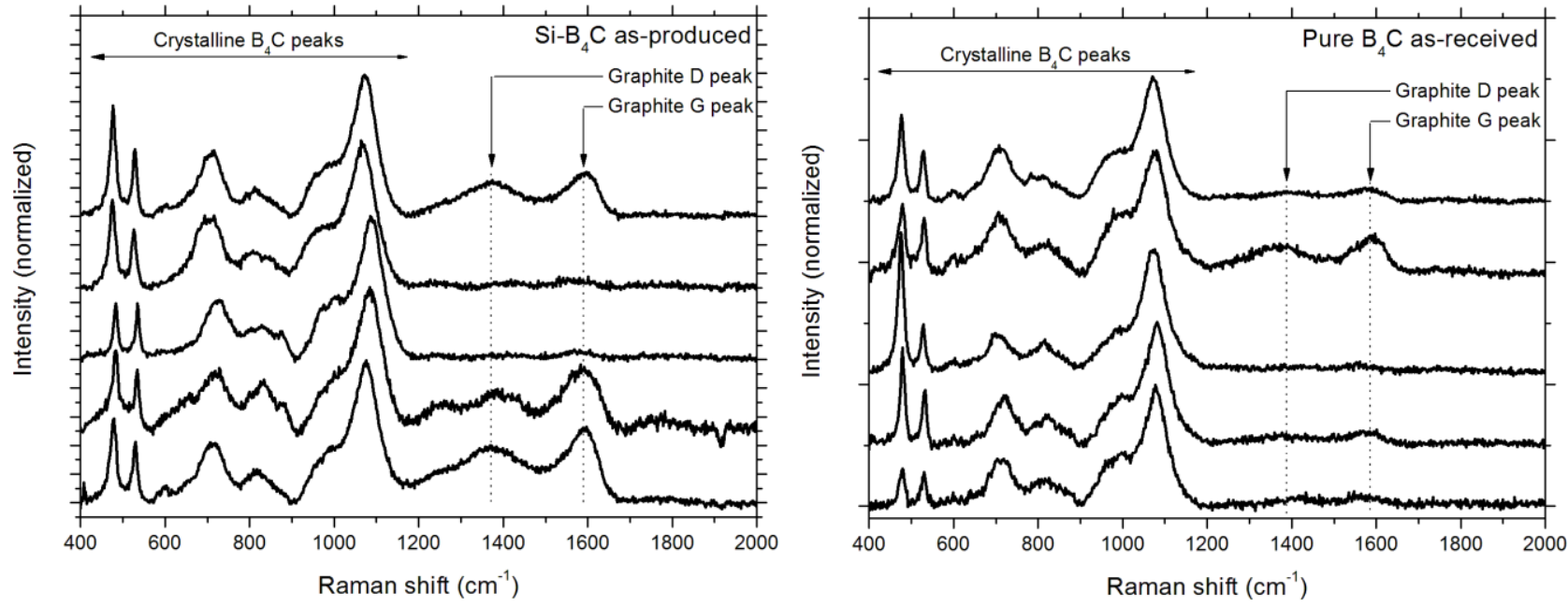

Fig. S1. Raman spectra of pure and silicon-doped boron carbide as received/produced. Each spectrum was collected from a different location on the sample. Peaks from $450-1200 \mathrm{~cm}^{-1}$ originate from crystalline boron carbide ${ }^{3,4}$, but in both pure and silicon-doped material Raman peaks at ca. $1340 \mathrm{~cm}^{-1}$ and ca. $1580 \mathrm{~cm}^{-1}$ that originate from graphite impurities ${ }^{19}$ are also present in some spectra. The amount of graphite impurity is greater in the silicon-doped sample.

\section{Additional raman spectra of pure and silicon-doped boron carbide following pressure-treatment}
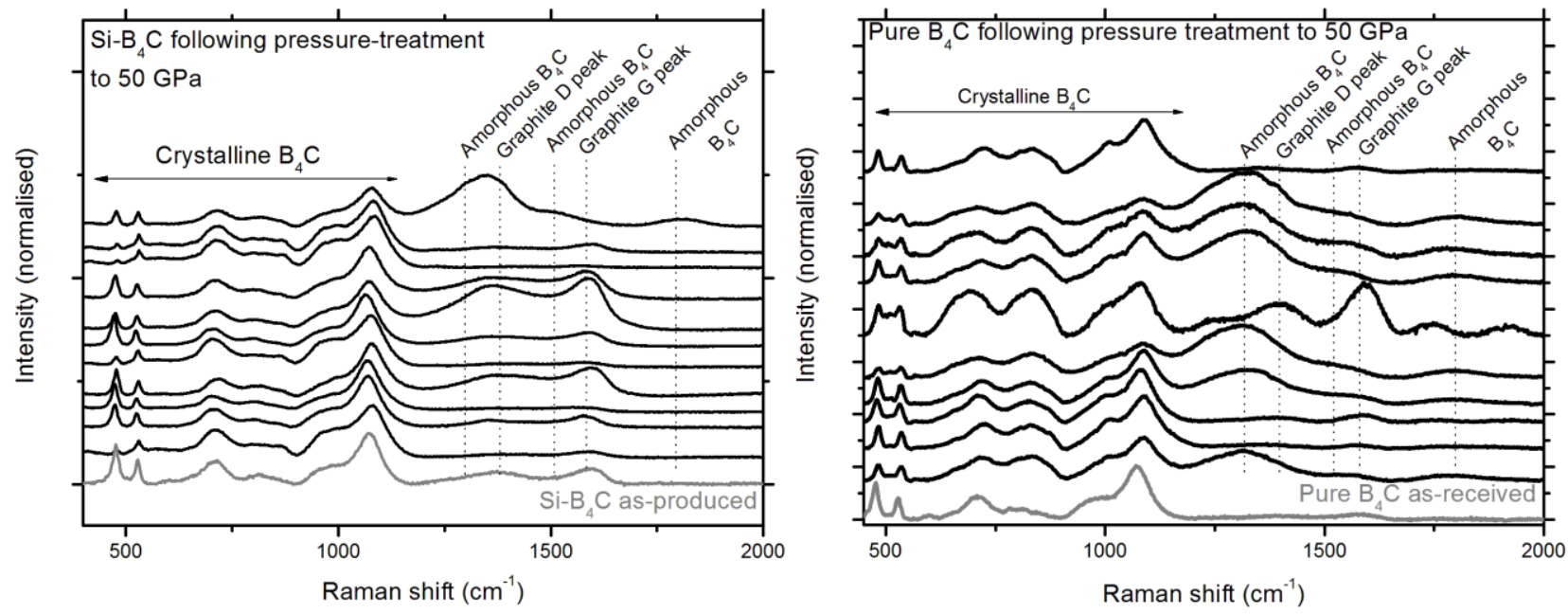

Fig. S2. Black lines - raman spectra of pure and silicon-doped boron carbide taken after pressure-treatment to $50 \mathrm{GPa}$. In each case, spectra were collected from 10 different locations in the sample chamber. 
Following pressure-treatment of the pure sample (bottom panel), new peaks at ca. $1325 \mathrm{~cm}^{-1}, 1520 \mathrm{~cm}^{-1}$ and $1810 \mathrm{~cm}^{-1}$ characteristic of amorphous boron carbide appear with significant intensity in $7 / 10$ of spectra collected. In the case of the silicon-doped sample (top panel), these peaks appear in only $1 / 10$ of the spectra collected - indicating a far higher level of amorphization in the pure sample. Grey lines representative spectra of samples before pressure treatment for comparison.

Note that we observe different Raman spectra from different parts of the sample in all cases, both before and after pressure treatment. Just like pure boron carbide, our samples of silicon-doped boron carbide asproduced are not spatially homogeneous. This is well documented for the case of pure boron carbide; samples as-produced can contain areas of different polytypes and free carbon impurities (ref. 3 and citations therein).

In the case of the spectra collected after pressure-treatment the nature of the experiment provides additional cause for the observation of different Raman spectra from different areas of the sample, again in agreement with the findings of previous studies on pure boron carbide (see, for instance, ref. 4). We expect different polytypes to collapse at different pressures. Also, when conducting an experiment to relatively high pressure on a hard material such as B4C under non-hydrostatic conditions (i.e. no pressure-transmitting medium) it is expected that pressure will vary by up to $\pm \sim 2$ GPa throughout the sample chamber and that shear stresses will be present. Therefore, not all areas of the sample will experience identical stress conditions.X-ray

\section{diffraction analysis of pure and silicon-doped boron carbide before and after pressure-treatment to $50 \mathrm{GPa}$}
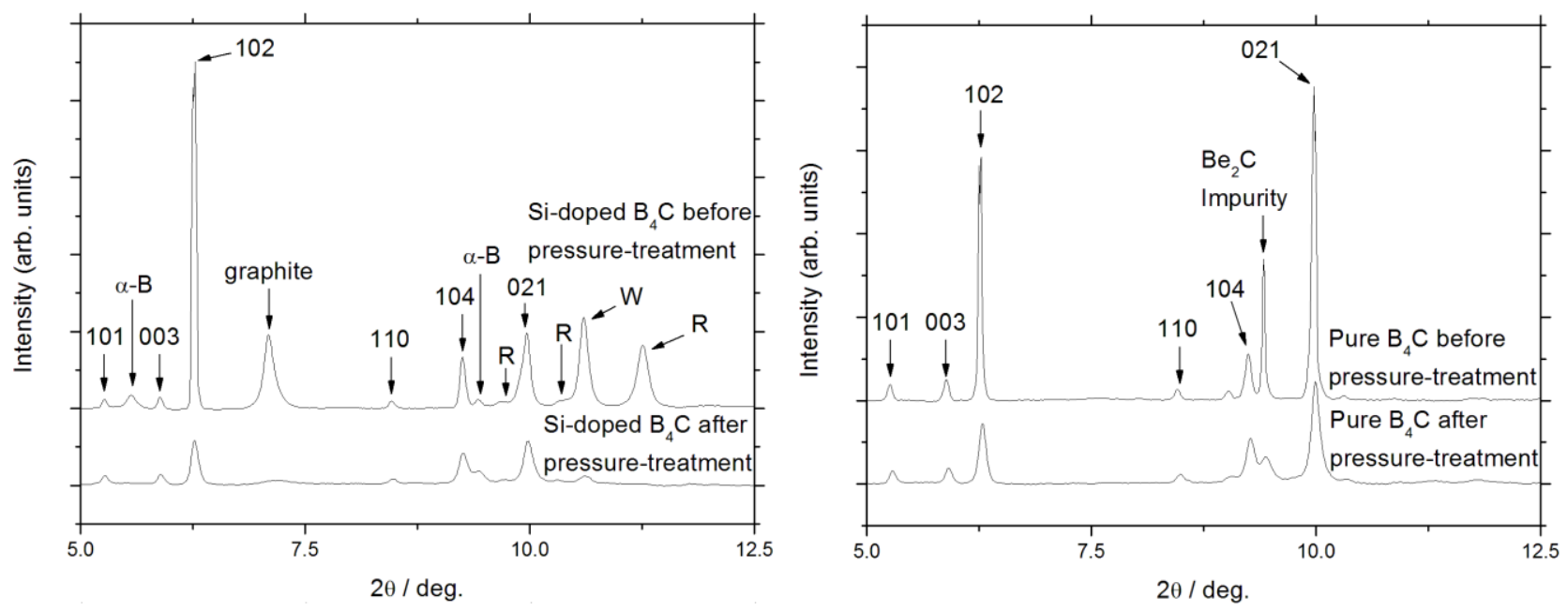
Fig. S3. X-ray diffraction patterns of silicon-doped (top panel) and pure (bottom panel) boron carbide before and after pressure-treatment to $50 \mathrm{GPa}$. On both panels indexed peaks are from boron carbide and other peaks are from impurities or the gasket of the diamond anvil cell. $\alpha-B-\alpha$-boron impurity (expected in silicon-doped boron carbide), graphite - impurity (expected in silicon-doped boron carbide), R - rhenium (gasket material in diamond anvil cell), W - tungsten (most likely small piece of needle used to load boron carbide into the diamond anvil cell), $\mathrm{Be}_{2} \mathrm{C}$ - impurity (expected in pure boron carbide).

For pure boron carbide the effect of pressure on the most intense X-ray diffraction peak (the 021 peak at $c a .10^{\circ} 2 \theta$ ) is an increase in the FWHM from $0.057^{\circ}$ to $0.115^{\circ}$. For the silicon-doped sample, the 021 peak is also subject to broadening but the change is less substantial. Prior to the application of pressure the FWHM is $0.107^{\circ}(0 \mathrm{GPa})$ but changes to $0.115^{\circ}(\mathrm{sic})$ following pressure treatment. Similar analysis of the 102 peak is possible: for pure boron carbide, the application of pressure increases the FWHM by approximately $100 \%$, for the silicon-doped sample the FWHM increases by approximately $40 \%$. See table of results in table S1.

\begin{tabular}{|c|c|c|c|c|c|}
\hline $\begin{array}{l}\text { Pure } B_{4} C \\
\text { sample }\end{array}$ & Index & $\mathrm{d} / \AA$ & $2 \theta /^{\circ}$ & FWHM $/^{\circ}$ & $\begin{array}{l}\text { Domain size } \\
\text { (Scherrer) })^{\star} / \mathrm{nm}\end{array}$ \\
\hline $\begin{array}{l}\text { Before } \\
\text { pressure }\end{array}$ & 021 & 2.365 & 9.966 & 0.057 & 240 \\
\hline $\begin{array}{l}\text { After } \\
\text { pressure }\end{array}$ & 021 & 2.374 & 10.04 & 0.115 & 81 \\
\hline
\end{tabular}

\section{Si-doped sample}

$\begin{array}{llllll}\text { Before } & 021 & 2.3799 & 9.963 & 0.107 & 147\end{array}$

pressure 
After

pressure

* The instrumental peak broadening was measured over a narrow angular range and modelled with a simple linear fit. Domain sizes were calculated for the samples including the instrumental broadening for several peaks. Mean values are reported.

Table S1. Table of parameters obtained from analysis of X-ray diffraction patterns of pure and silicon-doped boron carbide before and after pressure-treatment to $50 \mathrm{GPa}$. Broadening of boron carbide diffraction peaks following pressure treatment occurs in both cases, but is more severe for the pure sample.

\section{X-ray diffraction data collected in-situ at $50 \mathrm{GPa}$}

X-ray diffraction data was collected from pure and silicon-doped boron carbide in-situ in the DAC at high pressures up to $50 \mathrm{GPa}$. Evidence for structural collapse was not found. An X-ray diffraction pattern collected at $50 \mathrm{GPa}$ from silicon-doped boron carbide (figure S4) was fitted to obtain structural parameters a $=5.41(13) \AA, c=11.6(3) \AA$, Unit Cell Volume $=294 \AA^{3}$. These are in agreement with the known equation of state of pure boron carbide under hydrostatic conditions ${ }^{26}$. Further detailed analysis of the X-ray diffraction patterns collected in-situ in the DAC was not attempted due to the poor quality of the raw data. The extremely non-hydrostatic conditions created by compressing a super-hard material to $50 \mathrm{GPa}$ with no pressure-transmitting medium result in the poor signal-to-noise ratio observed in figure $\mathrm{S} 4$ and also result in spatial variation of pressure within the DAC by as much as $\approx \pm 2 \mathrm{GPa}$ so the collection of high quality X-ray diffraction data is not possible. The situation is exacerbated by the fact that as low-Z elements boron and carbon have a small X-ray scattering cross-section. 


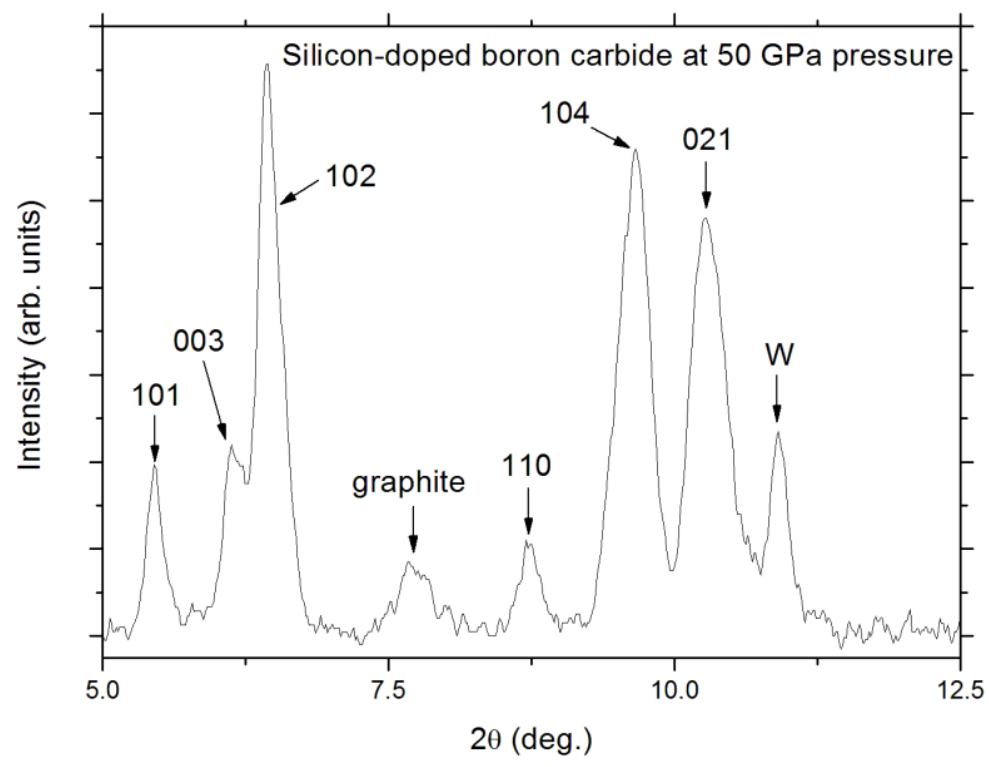

Fig. S4. X-ray diffraction pattern collected from silicon-doped boron carbide in-situ in the DAC at 50 GPa. Note poor signal-to-noise ratio compared to patterns collected at ambient conditions (figure S3). W tungsten. 


\section{Additional TEM images}

Both pure and silicon-doped samples were studied extensively following pressure-treatment with high-resolution transmission electron microscopy. Figure S5 shows typical low-magnification images and Figure S6 shows typical high-magnification images. Many further images were taken and large areas of both samples were surveyed.
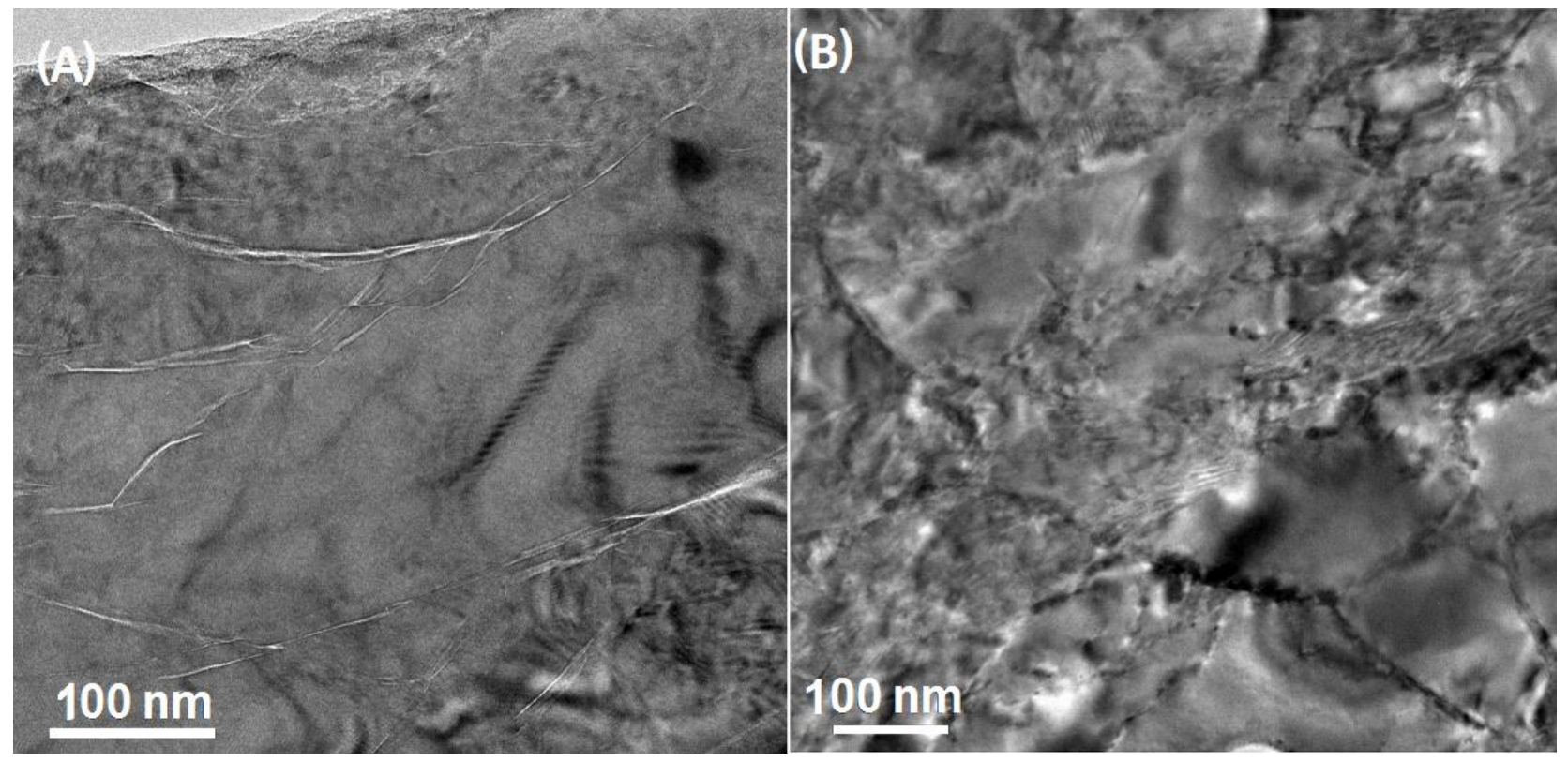

Fig. S5 Low-magnification TEM images following pressure-treatment of $(A)$ pure boron carbide showing significant micro cracking and $(B)$ silicon-doped boron carbide with no cracking in evidence but significant contrast from bending probably arising from residual stresses. 

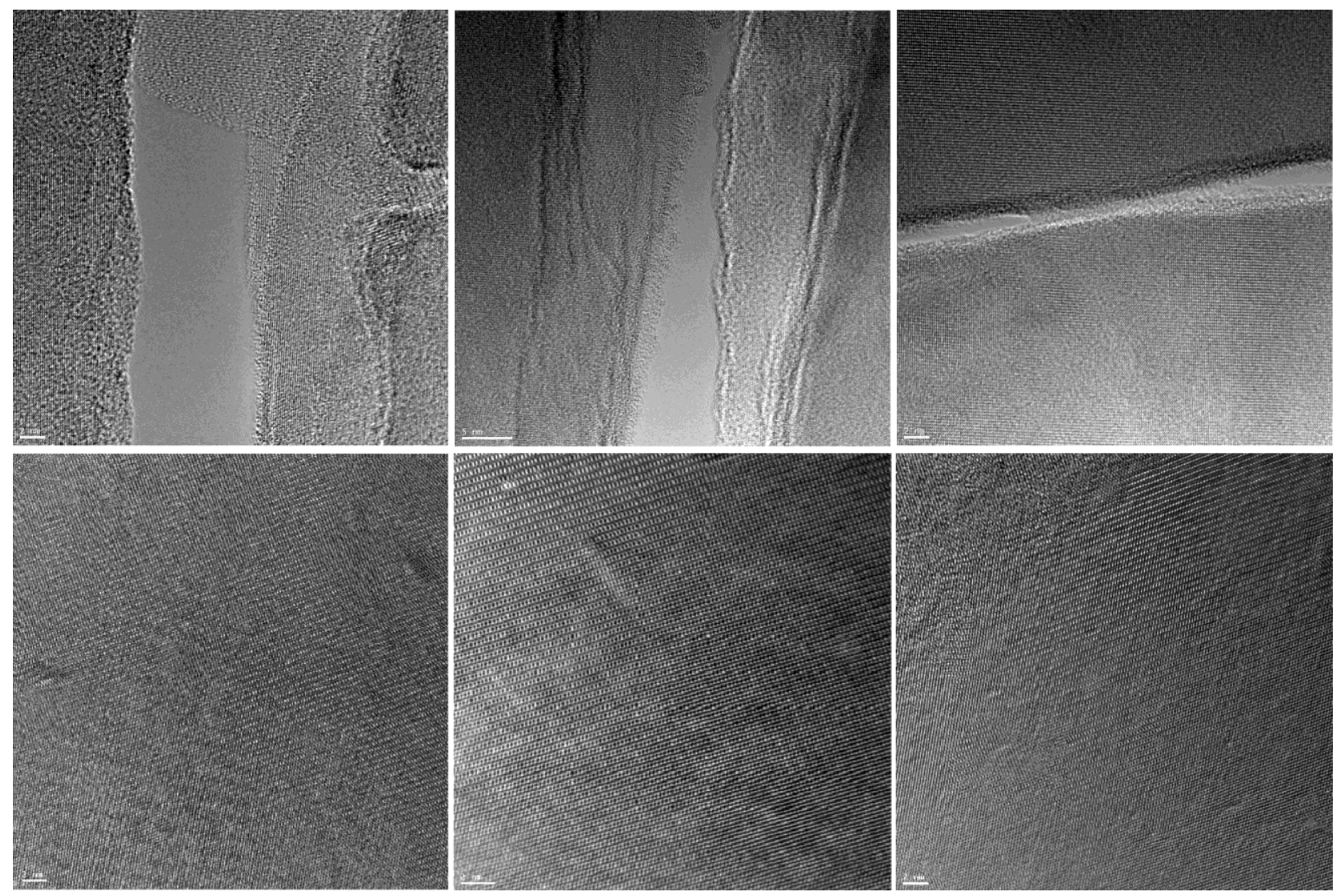

Fig. S6. High-magnification TEM images of pure (top panel) and silicon-doped (bottom panel) boron carbide following pressure-treatment to $50 \mathrm{GPa}$. Localized amorphous bands $2-10 \mathrm{~nm}$ across were frequently observed in the pure material, but were not observed in the silicon-doped material. Scale bars are $2 \mathrm{~nm}$, except for top panel centre image which is $5 \mathrm{~nm}$.

\section{Prediction of polytype concentrations as a function of silicon content and synthesis temperature}

Fanchini et al. (refs. 11 and 12) calculated the Gibbs free energy of the different possible polytypes in boron carbide using density functional theory. Using these results, it is possible to predict the concentrations of the different polytypes as a function of synthesis temperature as follows. During synthesis, different (microscopic) parts of the material experience different energies E, with probability distribution $f(E)$ :

$$
f(E) \propto e^{-\frac{E}{k T}}
$$


All polytypes are therefore synthesized with different concentration $f_{i}=f\left(G_{i}\right)$ where $G_{i}$ is the Gibbs free energy of the polytype in question. Fanchini et al. calculated the Gibbs free energies of all relevant polytypes in pure boron carbide ${ }^{11}$. Using this data, it is possible to calculate $f_{i}$ for all polytypes and hence normalise the values of $f_{i}$ to obtain the concentrations of the different polytypes as a function of synthesis temperature. In pure boron carbide, the difference in Gibbs free energy between the stable polytype $\left(\mathrm{B}_{11} \mathrm{C}_{\mathrm{p}}(\mathrm{CBC})\right)$ and the most stable minority polytype $\left(\mathrm{B}_{12}(\mathrm{CCC})\right)$ is predicted to be just $0.015 \mathrm{eV}$. This is predicted to increase drastically as silicon content is increased $^{12}$. For our samples (average silicon content of 1.2 at. \%) we expect the gap between the Gibbs free energy of the $\mathrm{B}_{12}(\mathrm{CCC})$ polytype to decrease such that the gap between it and that of the stable $\mathrm{B}_{11} \mathrm{C}_{\mathrm{p}}(\mathrm{CBC})$ polytype is $0.038 \mathrm{eV}$.

We can calculate $f_{i}$ for all polytypes for both pure and silicon-doped samples as a function of growth temperature. Figure $S 7$ shows the predicted concentrations of the $B_{11} C_{p}(C B C)$ and $\mathrm{B}_{12}(\mathrm{CCC})$ polytypes as a function of growth temperature for pure and 1.2 at. \% silicon-doped boron carbide.

The formation of a limited amount of phase separated boron and graphite is probable during boron carbide synthesis (we expect the Gibbs Free Energy for phase separated boron and graphite to be close to that for the $\mathrm{B}_{11} \mathrm{C}(\mathrm{CBB})$ polytype of boron carbide $\left.{ }^{11}\right)$. The formation of phase separated boron and graphite is therefore accounted for in the calculations above. However, it is important to note that the boron and graphite content actually observed in boron carbides is increased by the presence of leftover reactants from synthesis. 


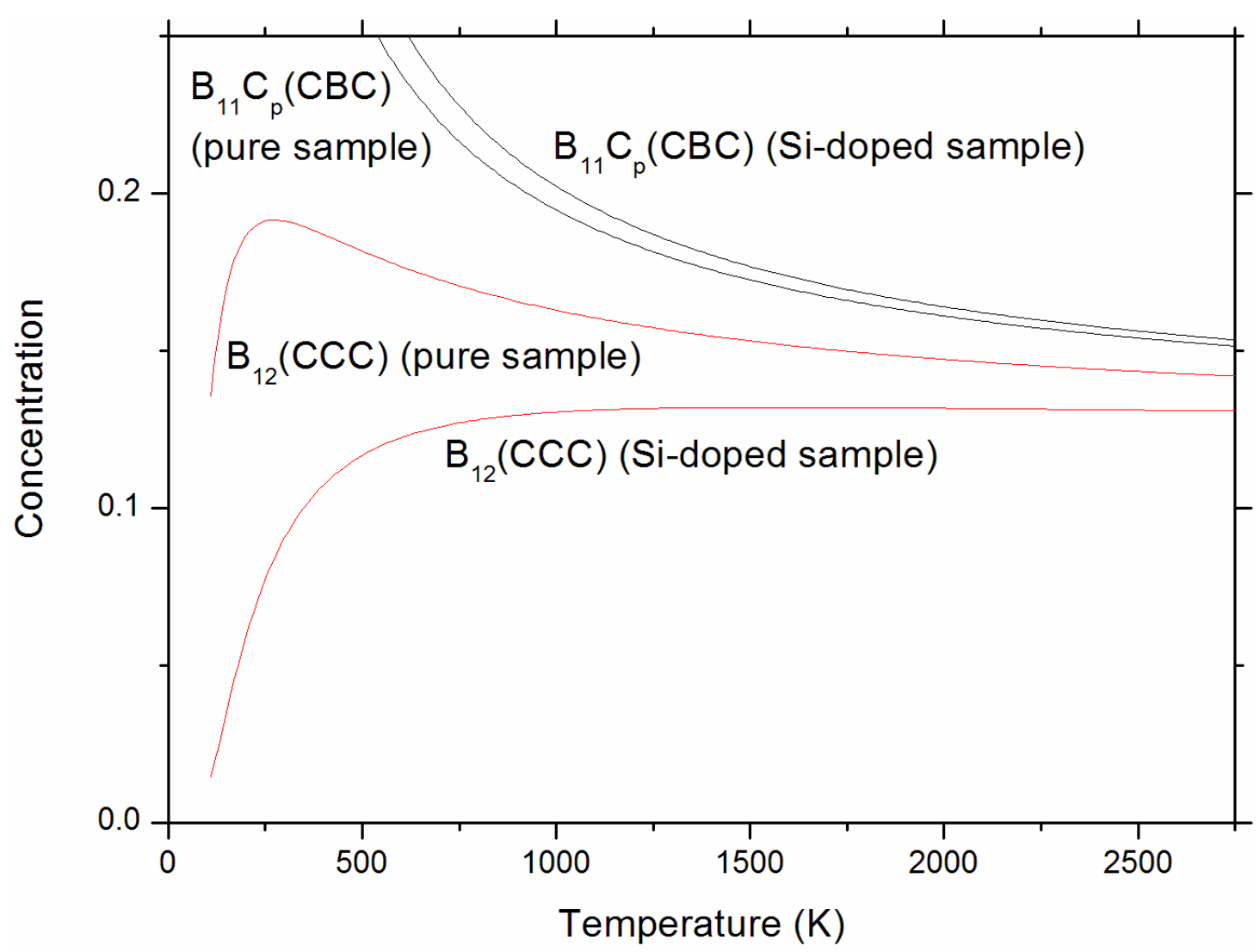

Fig. S7. Predicted concentration of $B_{11} C_{p}(C B C)$ and $B_{12}(C C C)$ polytypes as a function of growth temperature for pure and 1.2 at. \% silicon-doped boron carbide.

The temperature range of interest ranges from $1423 \mathrm{~K}$ (synthesis temperature for our silicon-doped boron carbide samples) to $2223 \mathrm{~K}$ (synthesis temperature for pure boron carbide ${ }^{17,18}$ ). From Fig. S9 we can see that in this temperature range the concentration of the $\mathrm{B}_{12}(\mathrm{CCC})$ polytype is expected to vary little with growth temperature. As growth temperature is decreased in this region, $\mathrm{B}_{12}(\mathrm{CCC})$ concentration actually increases (more so for the pure sample). This may seem counter-intuitive at first given that the $\mathrm{B}_{12}(\mathrm{CCC})$ polytype has a higher Gibbs free energy than the stable $\mathrm{B}_{11} \mathrm{C}_{\mathrm{p}}(\mathrm{CBC})$ polytype. It happens because, as temperature is decreased from ca. $2750 \mathrm{~K}$ to ca. $1500 \mathrm{~K}$ the $\mathrm{B}_{12}(\mathrm{CCC})$ polytype forms in preference to other, even less stable polytypes (e.g. $\mathrm{B}_{11} \mathrm{C}_{\mathrm{p}}(\mathrm{CCB})$ ). Only at much lower temperatures (lower than ambient temperature) can synthesis temperature alone be expected to reduce $\mathrm{B}_{12}(\mathrm{CCC})$ concentration in pure boron carbide. 